\title{
CIRCULATION ET TRANSMISSION DES SAVOIRS ISSUS DES PATRIMOINES IMMATÉRIELS : LE CAS AFRO-BRÉSILIEN
}

Christine Douxami

Presses de Sciences Po (P.F.N.S.P.) | « Autrepart »

$2017 / 2 \mathrm{~N}^{\circ} 82$ | pages 51 à 67

ISSN 1278-3986

ISBN 978272465355

Article disponible en ligne à l'adresse :

https://www.cairn.info/revue-autrepart-2017-2-page-51.htm

\section{Pour citer cet article :}

Christine Douxami, « Circulation et transmission des savoirs issus des patrimoines immatériels : le cas afro-brésilien », Autrepart 2017/2 (N 82), p. 51-67.

DOI 10.3917/autr.082.0051

Distribution électronique Cairn.info pour Presses de Sciences Po (P.F.N.S.P.).

(C) Presses de Sciences Po (P.F.N.S.P.). Tous droits réservés pour tous pays.

La reproduction ou représentation de cet article, notamment par photocopie, n'est autorisée que dans les limites des conditions générales d'utilisation du site ou, le cas échéant, des conditions générales de la licence souscrite par votre établissement. Toute autre reproduction ou représentation, en tout ou partie, sous quelque forme et de quelque manière que ce soit, est interdite sauf accord préalable et écrit de l'éditeur, en dehors des cas prévus par la législation en vigueur en France. Il est précisé que son stockage dans une base de données est également interdit. 


\title{
Circulation et transmission des savoirs issus des patrimoines immatériels : le cas afro-brésilien
}

\author{
Christine Douxami*
}

Au Brésil, les groupes dits «minoritaires » - amérindiens et afro-brésiliens sont engagés depuis les années 1930 dans la revalorisation des pratiques et des savoirs de leurs communautés. Dans les années 2000, ils se sont emparés des plans de sauvegarde de leur patrimoine immatériel lancés par différents acteurs transnationaux comme l'Organisation des Nations unies pour l'éducation, la science et la culture (Unesco). Cette dernière souligne le bénéfice de l'adoption de la convention du patrimoine immatériel, tant pour les pays du Nord que du Sud : "Cette transmission du savoir a une valeur sociale et économique pertinente pour les groupes minoritaires comme pour les groupes sociaux majoritaires à l'intérieur d'un État, et est tout aussi importante pour les pays en développement que pour les pays développés » [Unesco, sans date]. Elle définit le Patrimoine culturel immatériel (PCI) dans la convention pour la sauvegarde du patrimoine culturel immatériel d'octobre 2003, comme une entité mouvante :

«On entend par "patrimoine culturel immatériel" les pratiques, représentations, expressions, connaissances et savoir-faire - ainsi que les instruments, objets, artefacts et espaces culturels qui leur sont associés - que les communautés, les groupes et, le cas échéant, les individus reconnaissent comme faisant partie de leur patrimoine culturel. Ce patrimoine culturel immatériel, transmis de génération en génération, est recréé en permanence par les communautés et groupes en fonction de leur milieu, de leur interaction avec la nature et de leur histoire, et leur procure un sentiment d'identité et de continuité, contribuant ainsi à promouvoir le respect de la diversité culturelle et la créativité humaine. Aux fins de la présente Convention, seul sera pris en considération le patrimoine culturel immatériel conforme aux instruments internationaux existants relatifs aux droits de l'homme, ainsi qu'à l'exigence du respect mutuel entre communautés, groupes et individus, et d'un développement durable » [Unesco, 2003, article 2].

Pour l'Unesco, le PCI touche différents domaines qui sont tous liés à des cultures vivantes considérées comme sources de renouveau des sociétés contemporaines. Il est garant du maintien de la diversité culturelle et d'un sentiment

\footnotetext{
* Anthropologue, membre titulaire de l'IMAF-UMR 8171 (CNRS)/243 (IRD) et MCF HDR en arts du spectacle à l'université de Franche-Comté.
} 
d'appartenance sociale, et par conséquent, générateur d'un développement politique et économique durable. Lorsque les politiques publiques nationales s'attachent à la création de plans de sauvegarde de savoirs issus des patrimoines immatériels auparavant niés, car considérés comme minoritaires ou subalternes, elles créent pour ces savoirs une nouvelle légitimité dans la société dans laquelle elles s'insèrent, en se détachant lexicalement de ce qui se pratiquait auparavant (savoirs populaires, folkloriques) et en se référant à un « label » internationalement reconnu.

Ce label « patrimoine immatériel »n'est pas politiquement neutre puisqu'il met en jeu les identités nationales (en permanente élaboration) et la construction de la mémoire collective à l'aune d'un regard transnationalisé. Nora, dans son ouvrage Les Lieux de mémoire, est particulièrement attentif à cette question qui constitue l'un des enjeux du débat autour du concept de patrimoine culturel immatériel [Nora, $1984 ; 1986 ; 1992]^{1}$. Différents sens peuvent être appliqués aux concepts d'identité, d'histoire et de mémoire dans la définition du PCI, en fonction du contexte ethnique ou national au sein d'un monde contemporain globalisé. De nombreux auteurs, tant au Brésil qu'en Europe se sont penchés sur les ambiguïtés du PCI telles que l'Unesco les définit [Nas, 2002 ; Claessen, 2002 ; Jeudy, 2001 ; Jolher, 2002 ; Stoczkowski, 2009 ; Leimgruber, 2010 ; Bortolotto, 2011 ; Capone, Ramos de Morais, 2015 ; Tornatore, 2010 ; Sant'Anna, 2001 ; Telles, 2007 ; Alencar, 2010 ; Taylor, 2008 ; Smith, Akagawa, 2008; Smith, 2006]. Mais les questions suscitées par le PCI préexistent à la notion elle-même, tout comme les politiques publiques qui leur ont été associées [Abreu, Chagas, 2003 ; Belas, 2004 ; Gonçalves, 1995 ; Telles, 2007]. Elles se réfèrent à la place des productions culturelles des populations catégorisées comme subalternes - pour des raisons sociales, raciales, politiques, économiques au sein des États-nations [Cavalcanti, 2001]. Elles interrogent de nouveau la classification de la production issue des classes dites subalternes à l'intérieur d'une hiérarchisation sociale de ce qui est considéré, ou non, comme «artistique ». Historiquement, la classification de ces objets ou de ces manifestations culturelles a relevé d'un choix idéologique marqué par les conquêtes coloniales.

Nous verrons ici que la notion de PCI telle que diffusée par l'Unesco ne répond pas à la question cruciale du statut artistique de ces manifestations. Elle permet la valorisation «en bloc » de ces expressions culturelles en se détachant d'un débat épistémologique et idéologique qui reste cependant sous-jacent. Cette controverse ressort très nettement dans la société au moment de l'application des plans de sauvegarde, comme nous le voyons pour le Brésil où les maires « blancs » issus des élites traditionnelles refusent souvent de collaborer à la mise en place de plans de sauvegarde de patrimoines immatériels afro-brésiliens, leur refusant tout statut d'œuvre d'art [Douxami, 2015] ${ }^{2}$. D'ailleurs, le gouvernement Temer,

1. Au sujet de la construction de la mémoire et de l'oubli à l'échelle de la nation, voir Ciarcia Gaetano [2016].

2. Ce qui nous intéresse ici est cette puissance fédératrice de la notion de patrimoine immatériel qui permet, par le biais de plans de sauvegarde, la valorisation concrète de pans entiers de la société et de leurs productions de savoirs spécifiques. En ce sens, les plans de valorisation du patrimoine immatériel sont 
composé exclusivement d'hommes blancs, a supprimé dès son arrivée en 2016 le ministère de la Culture ${ }^{3}$ et celui des Femmes, de l'Égalité raciale et des Droits humains et tente d'inverser le processus de reconnaissance des PCI issus des minorités mis en place par le Parti des travailleurs. Se joue au Brésil, à travers le PCI, la question de la reconnaissance de la place des populations dites minoritaires au sein d'un discours de construction nationale.

Nous traiterons donc, dans un premier temps, de la complexité du statut artistique des savoirs issus des patrimoines immatériels. Puis, dans un second moment, nous aborderons comment s'effectuent la reconnaissance du savoir issu du PCI et la transmission par «l'éducation patrimoniale». Enfin, nous traiterons du lien entre création artistique et diffusion-transmission du PCI.

\section{La complexité du statut artistique des savoirs issus des patrimoines immatériels}

À la suite d'un long débat datant des années 1930 autour de ce que serait un « objet ethnographique », Jean-Marie Shaeffer interroge le statut des objets du musée du Quai Branly issus, pour leur majorité, de sociétés non occidentales. Il s'interroge sur le fait qu'ils n'aient souvent pas accès au statut d'œuvres d'art ; or ceci recoupe les questions plus amples posées sur le statut des savoirs issus des patrimoines immatériels. Pour Shaeffer, il faut préalablement distinguer la réaction du spectateur face à l'objet présenté (sa réception esthétique) et la volonté du créateur au moment de réaliser sa production (sa création artistique). Il met l'accent sur la nécessité de dissocier l'acte cognitif d'attention du spectateur de l'acte créatif. Un objet peut être réalisé sans aucune velléité artistique et provoquer pourtant une émotion esthétique chez le spectateur. Ainsi, au Brésil, les participants des Autos (danse-théâtre liée à une liturgie religieuse, catholique initialement) se considèrent comme des «joueurs» brincantes, ils déclarent qu'ils «s'amusent» et non comme des artistes et pourtant le spectateur peut vivre une émotion artistique ${ }^{4}$. Shaeffer [2008] s'exprime en ces termes :

«Bien que sous le terme d'“esthétique" nous englobions souvent la création des œuvres et leur "contemplation", il faut, bien entendu, dissocier les deux. On peut rappeler à ce propos qu'Alexander G. Baumgarten (l'inventeur de la notion) avait défini le terme "esthétique" explicitement en termes d'attention cognitive et non pas en termes de création (de tecknè). Kant, de même, avait insisté sur le fait que la relation esthétique ne se définissait pas par un certain type d'objets, mais par une visée attentionnelle particulière. Il importe plus que jamais de revenir à ces conceptions : si l'on ne distingue pas d'entrée de jeu la problématique esthétique

éminemment politiques et dépendent d'une vision globale de l'État-nation concerné et ont un impact concret tant sur la vie des détenteurs de ce savoir immatériel que sur la transmission de ce savoir. Au sujet du rapport du politique à la nation voir [Bortolotto, $2007 ; 2011$, particulièrement l'introduction de son ouvrage collectif ; Capone, Ramos de Morais, 2015 ; Goncalves, 2002 ; Penteado, 2015 ; Douxami, 2015].

3. Le ministère de la Culture a été rétabli à la suite de l'occupation des différents lieux, mais non celui des Femmes, de l'Égalité raciale et des Droits humains.

4. Pour une définition des Autos, voir Douxami [2010]. 
de la problématique artistique, on se condamne à une confusion analytique majeure. D'abord, les ressources mentales mises en œuvre dans un acte de création et dans un acte d'attention ne coïncident pas. Ensuite, la relation esthétique n'implique aucune contrainte objectale : n'importe quoi peut être investi esthétiquement. De même, la création artistique n'implique pas par essence une finalité esthétique. Enfin, la réception d'une œuvre d'art, quelle qu'ait été la finalité de cette création, n'est pas nécessairement de nature esthétique. » [Shaeffer, 2008, p. 173]

Pour permettre d'avancer dans ce débat et pour faire suite à l'impossible définition de ce que serait une « œuvre d'art», Schaeffer [2008] propose la notion de « signal à coût élevé » :

«Mon hypothèse est que les "œuvres d'art" constituent une des cristallisations d'une classe plus vaste d'entités et d'événements qui renvoient à une constante fonctionnelle transculturelle et transhistorique, à savoir ce qu'en biologie et en sociologie on appelle la production de signaux à coût élevé. Par signal à coût élevé, on entend tout signal qui prend le contre-pied du principe d'économie régulant en général la signalisation humaine et animale. [...] Les signaux coûteux d'ordre artistique se rencontrent surtout dans des situations où les humains, que ce soit comme individu ou comme groupe, entrent en rapport avec une altérité, par exemple avec des esprits ou avec les ancêtres ou les morts, et plus fondamentalement encore, lorsqu'ils se trouvent face à l'énigme de leur propre identité existentielle à l'intérieur du monde social, naturel et cosmique, etc. Bref, chaque fois que nous sommes pris dans un réseau interhumain et cosmique qui ne va pas de soi et qui nous amène à nous interroger sur nous-mêmes » [Shaeffer, 2008, p. 174×175]

À partir de cette amplification de ce que serait une œuvre d'art selon Shaeffer qui renverse la définition des «Beaux-Arts » telle que crée à la Renaissance, Carlo Célius, en vient à déterminer ce qui serait de l'ordre de «domaines de créations plastiques » et qui correspondrait à différentes matérialisations de la «cristallisation » comme elle est définie par Shaeffer. Pour Carlo Célius [2015] :

«La création plastique comprendrait une classe d'objets résultant de la matérialisation de l'imaginaire à partir de quelques éléments de base, dont le trait (le dessin), la forme, la couleur et le volume. Le traitement technique de ces éléments, leur combinaison, les matériaux utilisés, les types d'objets qui en résultent, leurs fonctions, leur mode de valorisation varient d'une société à une autre, d'une époque à l'autre et, possiblement, d'une entité sociale à l'autre, dans une même société pour une période déterminée. Ce sont ces formes d'organisation des pratiques de création plastique que j'appelle domaines de création. C'est à ce niveau que se constituent des normes, des conventions, des genres ; c'est là que s'établissent la nature et les critères de jugement, que se définissent les types de discours, les fonctions des objets et leur mode de circulation. Les relations entre les domaines varient selon les périodes en raison de circonstances particulières. » [Célius, p. 111]

Dans le cas de Haïti, auquel se réfère Carlo Célius, cette notion de domaine de création permet d'inclure à la fois le domaine de création propre au Vaudou en tant que rituel et le domaine de création de l'acte artistique qui s'en inspire. Un PCI, quel qu'il soit, peut appartenir à un « domaine de création » tel que défini par Carlo Célius, en tant que « cristallisation d'un signal à coût élevé » et ce, de façon horizontale vis-à-vis d'autres domaines de création habituellement reconnus 
comme tels, car issus des « Beaux-Arts ${ }^{5}$. De même, certains créateurs, appartenant à d'autres domaines de création peuvent s'inspirer d'un PCI et l'inclure dans leur création artistique et participent à la diffusion des savoirs issus du dit PCI. Le candomblé a beaucoup été évoqué par la scène artistique brésilienne depuis le début du $\mathrm{XX}^{\mathrm{e}}$ siècle : qu'il s'agisse du domaine de création plastique ou chorégraphique, nous y reviendrons.

Dans le cas plus spécifique des arts de la scène, pour reprendre le cadre mis en place ci-dessus, il nous semble donc pertinent de déterminer la création scénique qui comprendrait une classe de représentations spectaculaires, correspondant à une matérialisation de l'imaginaire à partir de quelques éléments de base, dont la présence corporelle, le geste, l'adresse à un public. Il existerait alors plusieurs domaines de créations scéniques dans lesquels, pour le Brésil, les autos liturgiques seraient un domaine, les danses rituelles un autre, la chorégraphie contemporaine un autre encore, etc. Ceci permet d'éviter une hiérarchisation des manifestations.

La question du statut des savoirs touchant aux domaines de la création plastique issus des populations subalternes, notamment dans les sociétés postcoloniales, a été largement abordée, mais fort peu d'écrits ont traité des arts de la scène sur cette problématique, mis à part Victor Turner [1974 ; 1987] et Richard Schechner [1985 ; 1988] au sein des Performance studies américaines qui cherchent à valoriser, sans en spécifier toujours clairement les contours, un «autre théâtre ${ }^{6}$. Cependant, les problématiques se recoupent quant aux statuts de ces productions culturelles : comme il n'y a pas d'arts plastiques «primitifs » il n'existe pas de « préthéâtre », mais différents domaines de créations au sein d'une même société, sur un pied d'égalité. Sous cette optique, les PCI se rattachant tant au domaine de création plastique qu'à celui de la création scénique sont alors susceptibles d'obtenir une même reconnaissance que d'autres patrimoines. Ainsi, dans le cas du Brésil, l'Iphan (Instituto do patrimônio histórico e artistico nacional, organe relatif au patrimoine lié au ministère de la Culture brésilien) a pendant des années reconnu le patrimoine comme étant avant tout matériel (architectural notamment), et portait aux nues principalement l'architecture baroque qui correspondait aux goûts des élites brésiliennes [Capone, Ramos de Morais, 2015 ; Pinheiro Bressan, 2006 ; Veloso, 1992 ; Vianna, 2004 ; Chuva, 2009 ; Falcão 2001]. L'Iphan est aujourd'hui, avec la reconnaissance du PCI, dans l'obligation de mettre sur un pied d'égalité ces réminiscences architecturales baroques coloniales et des PCI tels que la Samba de Roda. L'enjeu soulevé ici traite de la «colonisation des imaginaires » [Célius, 2015] et de la posture ambiguë des élites nationales depuis les indépendances, qui cherchent à se distinguer des anciens colons tout en restant profondément attachés aux codes, notamment artistiques, de ces derniers.

Ainsi, une fois ces éléments clarifiés sur le plan épistémologique, nous pouvons utiliser la notion de «patrimoine immatériel » pour ce qu'elle est : un outil pour

5. Pour un complément autour d'Haïti, lire également Célius Carlo [2005].

6. Voir à ce sujet la revue: The Drama review dirigée par Schechner. 
la valorisation de domaines de créations généralement exclus de ce qui est de l'ordre des beaux-arts, car issus de classes subalternes. Les beaux-arts, tels que diffusés dans le monde et particulièrement pendant la période de la colonisation, correspondent, en effet, à des «éléments de l'appareillage symbolique de domination » [Célius, 2015]. Cet outil conceptuel qu'est le PCI passe par le biais d'un dispositif concret: les plans de sauvegarde des PCI qui viabilisent leurs transmissions.

\section{La reconnaissance du savoir issu du patrimoine immatériel $(\mathrm{PCI})$ et la transmission par « l'éducation patrimoniale »}

Outre la question artistique liée au concept de PCI qui complexifie la diffusion de son savoir, la notion de PCI est nécessairement ambivalente et a pu être comprise de façons très diverses quant à la mise en place des politiques publiques et des plans de sauvegarde y afférents. Les définitions prennent en compte des catégories telles que l'identité, la mémoire et la notion de communauté ${ }^{7}$. Or ces notions se situent dans un processus dynamique sujet à transformation. Comment cet état de flux permanent est-il envisagé par les politiques publiques au sein des plans de sauvegarde ? S'agit-il de figer une manifestation dans un état donné au moment de sa patrimonialisation ou au contraire de la vivifier par une nouvelle transmission des savoirs issus de ce PCI ? Comment se choisit le savoir au sein même d'une manifestation ? Par exemple: si le maracatu rural, danse-théâtre populaire afro-brésilienne de Pernambuco au Brésil, se fait depuis quelques années avec des lunettes de soleil et des basquets pour chaque figurant, les acteurs de la patrimonialisation vont-ils décider qu'il faut revenir à une certaine « authenticité » de la manifestation et retirer les lunettes et les basquets dans une optique « primitiviste »?

Choisir de valoriser, ou non, un PCI est un choix politique, éthique, dans une logique générale de «dominant dominé ». Or, qui détient le pouvoir de légitimation si ce n'est des «experts » issus de classes, a priori, dominantes ? Qui peut déterminer qu'un savoir est en voie de disparition, ce qui constitue une des consignes de l'Unesco quant à la patrimonialisation d'un bien immatériel ? A priori, la communauté détentrice du PCI devrait être à même de le faire. Cependant, pour mettre en place le dossier de demande de sauvegarde du PCI, la procédure est complexe, souvent en ligne sur internet et nécessite que les personnes concernées soient suffisamment organisées pour mener le processus à bien, qu'il s'agisse d'une demande de reconnaissance nationale (auprès de l'Iphan au Brésil ou internationale après de l'Unesco). Ainsi, la manifestation de danse-théâtre afro-amérindienne du Guerreiro de Alagoas avait été choisie comme PCI de l'État d'Alagoas par le ministère de la Culture brésilienne et le groupe Treme Terra Mundial da cidade de Atalaia devait être à la tête d'un ponto de cultura (le « point de culture » correspond au financement sur projet d'une association choisie comme

7. Au sujet de la communauté, voir Maguet [2011]. 
fer de lance de la culture pendant un temps donné, généralement quatre ans) [Barbosa, Calabre, 2011 ; Fonseca, 1997] ${ }^{8}$. Mais l'organisateur du groupe, Ozeas Bomfim, analphabète, nous expliqua en 2013 «qu'il n'y avait rien compris » et n'avait finalement rien reçu ${ }^{9}$.

Cependant, certains parviennent à s'approprier cet outil tant pour la reconnaissance de leur PCI que pour la bonne gestion de leur plan de sauvegarde et à terme de la réussite de la passation des savoirs. Comme l'explique Laure Emperaire, lorsque les populations Enawene-Nawe du Mato Grosso parviennent à une reconnaissance patrimoniale par l'Iphan en 2010 du rite Yaokwa, ils le font dans un objectif politique assumé. Ce rite, « au cours duquel un immense barrage temporaire de bois est construit pour une pêche collective [...] La mobilisation de l'instrument patrimonial avait pour ambition de faire reconnaître à l'échelle nationale la richesse culturelle portée par ce rituel et d'assurer sa sauvegarde face aux plans de construction de barrages sur le Juruena » (cf. Emperaire, note 8). Ici, le «dominé » s'empare du processus de patrimonialisation pour la sauvegarde du savoir présent dans cette région ${ }^{10}$. Les populations de cette région, organisées, au sein d'une problématique plus ample de revendication militante d'autochtonie, sont parvenues à leurs fins : éviter le barrage et se faire connaître. Ce type d'action se situe dans le cadre d'une société préalablement organisée et particulièrement auprès des groupes revendiquant une autochtonie comme les Amérindiens ou une reconnaissance au sein de la nation comme les Afro-brésiliens.

L'exemple de la Samba de Roda permet de comprendre les processus de fonctionnement d'un plan de sauvegarde d'un PCI et de sa transmission. La Samba de Roda du Reconcavo de Bahia a été reconnue par l'Iphan en 2004 et par l'Unesco en 2005 et se caractérise par une performance jouée, dansée et musiquée. Une partie de ses participants était issue des mouvements post-dictature de revendication identitaire afro-brésilienne [sur la Samba de Roda, voir Sant'Anna, Sandroni, 2007 ; Amoroso, 2009 ; 2011 ; Sandroni, 2011 ; 2010 ; Douxami, 2015 ; Asseba, 2015]. Ayant déjà une relative connaissance préalable des enjeux de pouvoirs liés aux politiques publiques, ces derniers se sont organisés en association autogérée [Asseba, 2015] leur permettant une grande autonomie de gestion créant ainsi une jurisprudence de gestion patrimoniale. En effet, les autres patrimoines immatériels brésiliens ont généralement une gestion mixte. Par exemple, le Cirio de Nazaré (à Belem) est géré par l'Église catholique et par le Département de la culture du Para, le Jongo du Sudeste était administré jusqu'en 2013 par l'université fédérale

8. La gestion de Gilberto Gil et l'invention des «points de culture » [pontos de cultura 4204 de 2005 à 2014 selon le site du ministère de la Culture] ont permis le développement de manifestations culturelles à travers des appels à projets ouverts. Sur les pontos de cultura, voir Douxami [2015].

9. Entretien avec Ozeas Bomfim, 2 février 2013, municipalité de Arapiraca.

10. Information fournie lors du panel «Nouveaux acteurs, vieilles stratégies ? Conflits, médiations, alliances et recompositions autour de grands aménagements du Sud » (S. Benabou, S. Duvail, C. Médard, O. Evrard, L. Emperaire, P. de Robert, Ph. Léna) aux journées d'étude « Mondialisation et nouvelles dynamiques Sud-Sud, le développement en question », IRD-université Paris-Diderot, les 6 et 7 octobre 2015. 
Fluminense (UFF) puis par l'Iphan ${ }^{11}$. Le Carimbó reconnu en septembre 2014 a été en contact avec l'Asseba pour suivre un modèle associatif.

L'Asseba a ainsi pu développer, certes avec des fonds publics et un appui technique et consultatif de l'Iphan, un réseau de quinze maisons (casas do samba) sur tout le territoire du Recôncavo, avec une maison principale à Santo Amaro. Ce réseau lui permet de mieux développer ses stratégies politiques et l'organisation de la passation de son savoir. Des réunions sont organisées par la maison principale à Santo Amaro et à une plus petite échelle, avec les participants de trois ou quatre «maisons de la samba ». En effet, chaque maison possède un référent de l'association, un budget de fonctionnement, une salle informatique reliée à internet et organise des ateliers de transmission. Les ateliers proposés dans ce cadre, le plus généralement aux enfants ou aux adolescents, parfois hebdomadaires, parfois occasionnels en fonction des possibilités financières et du calendrier des maîtres de Samba de Roda, sont des cours variés dont l'objectif touche à la fois à l'approfondissement de la connaissance de la Samba de Roda au travers d'enseignements de sa musique, de son chant, de sa danse, de la fabrication de ses instruments (la machete notamment, petite guitare traditionnelle de la Samba de Roda) et la prise en main des outils permettant la pérennisation de ce savoir.

Ces outils visent l'apprentissage de notions de production musicale, à la fois en ce qui concerne les multiples appels à projets (internationaux, nationaux, régionaux, municipaux, publics ou privés), la négociation des cachets des participants, mais également le passage à la scène qui implique un changement dans la forme de la manifestation avec des costumes, un son non acoustique, une chorégraphie distincte liée à l'éloignement du public. Un studio d'enregistrement à la maison principale de Santo Amaro permet une autoproduction de CDs pour les groupes et une diffusion du savoir exogène à la communauté. Un autre outil proposé aux détenteurs est l'accès à l'université, permettant par la suite une meilleure gestion $\mathrm{du}$ PCI, mais également une mobilité sociale ascendante pour ses participants.

\section{Création artistique et diffusion du patrimoine immatériel}

Certains jeunes issus de la communauté de la Samba de Roda se désignent à une carrière artistique. Par le biais d'ateliers organisés par la Casa do samba, les jeunes apprennent à jouer de la Samba de Roda. Certains deviennent parfois des musiciens professionnels d'autres types de musiques plus «commerciales ». En dehors du fait qu'ils déçoivent ce faisant quelque peu leurs maîtres de samba, le plan de sauvegarde du savoir patrimonial a - en un sens - bien fonctionné. À titre individuel, ils acquièrent, en effet, un métier, et d'autre part, ils n'abandonnent pas totalement la Samba de Roda et créent des formes musicales hybrides. En d'autres termes, ces jeunes introduisent, au sein même de la musique

11. Informations fournies par Edivaldo Bolagi, un des fondateurs de l'Asseba, entretien à Salvador le 4 février 2014. 
contemporaine, des thèmes musicaux issus de la Samba de Roda, qu'ils croisent avec ceux de la musique actuelle.

Or il s'agit d'une démarche que nous avons évoquée plus haut : l'appropriation, par les artistes - originaires ou non de la manifestation de PCI - des différentes formes musicales, chorégraphiques et théâtrales issues des manifestations du PCI au sein de créations artistiques exogènes à la manifestation. Il existe un phénomène d'aller-retour entre des artistes endogènes à la manifestation qui introduisent leur art au sein de créations contemporaines et des artistes exogènes à la manifestation du PCI qui s'inspirent de ce dernier dans leurs pratiques artistiques, au sein d'autres «domaines de création artistiques » pour reprendre le schéma de Carlo Célius évoqué plus avant.

Cette tendance a été initiée dès les années 1920-1930 dans de nombreux pays d'Amérique latine et des Caraïbes, dans la volonté de créer, à la suite des récentes indépendances, un art national nourri aux sources indigènes et populaires. Des poètes, plasticiens, metteurs en scène, chorégraphes cherchèrent dans ces manifestations, une source d'identité et d'inspiration à même de donner une « originalité nationale et personnelle » à leurs travaux artistiques, tout en participant à la transmission et à la valorisation de ces dernières.

Dès les années 1920, il exista concomitamment un élargissement du champ de l'art, plus particulièrement avec le mouvement Dada qui «libère » l'artiste et « l'autorise » à se pencher sur d'autres matériaux, qu'il soit plasticien ou metteur en scène. De plus, des mouvements, tels que le primitivisme, ont parallèlement intégré d'autres horizons culturels dans leurs œuvres et ont décloisonné l'art. Les artistes du Sud souvent au contact de leurs pairs du Nord par le biais de différents « allers-retours » transocéaniques, incorporent cette transformation au cœur même de la définition, imposée à l'époque coloniale, de ce qui serait ou non, une « œuvre d'art ». On l'a vu, la notion d'œuvre d'art qui serait réservée à l'art des colonisateurs est remise en question [Célius, 2015]. Ainsi, Blaise Cendrars, immergé dans la diffusion de «l'art nègre » en France, partage ces idées avec Mario de Andrade [2002], futur instigateur du mouvement « anthropophagique » au Brésil et surtout précurseur dans la législation de reconnaissance et de valorisation de l'art populaire et du folklore dans les années $1930^{12}$. Mario de Andrade était d'ailleurs en lien avec son homologue haïtien, Jean Price-Mars, comme le soulignent Normélia Parise [2014] et Myriam Mompoint [2008].

À cette même période, en Europe, des anthropologues, pionniers, à l'instar de Michel Leiris, Marcel Griaule, Georges Balandier, puis de Jean Laude [2006], croisent des données dites ethnographiques et artistiques, donnant une nouvelle lecture des savoirs issus des patrimoines rencontrés lors d'expéditions de terrain [Sylla, 1999, p. 302]. Pourquoi, comme le rappelle Jean-Loup Amselle, « un objet

12. L'action de Mario de Andrade est comprise jusqu'à aujourd'hui comme le point de départ de la réflexion brésilienne autour du patrimoine culturel immatériel, qui a permis au Brésil de mettre en place une législation favorable dès sa constitution de 1934 . 
ethnographique serait-il dénué d'esthétique ${ }^{13}$ ? Jean-Loup Amselle interroge l'idée même «d'objet ethnographique », lui-même très discutable comme nous l'avons envisagé avec Shaeffer [2008]. Dès les années 1970, Michel Leiris contestait le statut primitiviste réservé à «l'objet ethnographique », au sein du musée de l'Homme, mais également plus généralement dans le milieu du marché de l'art. Il dénonce le fait que «l'objet ethnographique » ne soit envisagé que comme le « témoin d'un mode de vie » et ne puisse acquérir un statut d'œuvre d'art.

Le processus de valorisation du PCI par l'Unesco en 2003 a relancé ce questionnement d'inclusion, ou non, du savoir artistique issu du PCI au sein des créations artistiques contemporaines, et ce, dans un marché de l'art mondialisé dans lequel les stéréotypes de «l'ethnique » et de « l'authentique » apparaissent comme des critères à la fois de valeurs ou de non-valeurs tant symboliques que marchandes. Qu'il s'agisse d'un pas dans une chorégraphie, d'un personnage dans une dramaturgie ou d'un thème dans une œuvre plastique, le PCI devient potentiellement « incorporable » dans une œuvre contemporaine, modifiant par effet de réflexion le statut même du PCI appartenant potentiellement au domaine de l'art. De plus, ces œuvres participent à la transmission du savoir issu du PCI par le biais de la scène artistique.

Lorsque le curateur de la biennale de Bahia de 2015, Ayrson Heraclito, décide d'inviter des maîtres de manifestations de patrimoines immatériels dans le musée d'Art moderne de Bahia, les nommer performeurs, leur permettre de donner des ateliers aux côtés d'autres artistes chorégraphes performers et les placer en ouverture de la biennale, il s'agit d'un changement de statut de ce savoir dans cette ligne de reconnaissance initiée au début du $\mathrm{XX}^{\mathrm{e}}$ siècle. Le savoir des détenteurs de PCI est alors transmis avec le même statut artistique que celui des autres artistes présents à la Biennale de façon horizontale, comme le suggère la nonhiérarchisation des «domaines de création » issus d'une même «cristallisation d'un signal à coût élevé ». Il n'est plus ni «primitif» ni «folklorique » ou « ethnographique » et il acquiert le statut d'art contemporain. De plus, c'est Edivaldo Bolagi, qui appartenait parallèlement à l'équipe dirigeante de l'Asseba (on l'a vu : l'association qui gère le plan de sauvegarde de la Samba de Roda), qui a chorégraphié la performance d'ouverture de la biennale du 29 mai 2014 avec 33 alabês (individus dans le candomblé chargés de chanter en hommage aux orixás) des principaux lieux de culte du candomblé (Terreiros) et avec la présence de la chanteuse Inaicyra Falcão fille de feu Mestre Didi, grand officiant du candomblé ${ }^{14}$. Ainsi, en étant inclus dans la Biennale, le chant des officiants du candomblé et les danses d'autres patrimoines immatériels acquièrent un statut

13. Intervention de J.-L. Amselle au séminaire "Supports et circulation des savoirs et des arts », EHESS, du 13 novembre 2015.

14. Stefania Capone et Mariana Ramos de Morais [2015] soulignent d'ailleurs qu'il reste étonnant que le candomblé soit patrimonialisé uniquement sous sa forme matérielle, les terreiros (les lieux de culte) et non sous sa forme immatérielle. Selon nous, lors de cette Biennale, il s'agissait de valoriser la tradition du candomblé sous sa forme immatérielle. 
d'œuvre d'art. La question du rapport entre art et religion tout comme celle du secret est, elle aussi, complexe, mais ne sera pas abordée ici.

Par ailleurs, des metteurs en scène et chorégraphes contemporains s'approprient des formes du PCI au sein de leur création ce qui devient une des possibilités de passation du savoir issu de ces manifestations. Ce dernier est transmis à d'autres publics, tant nationalement qu'internationalement, qui n'auraient pas, en temps normal, accès à ces manifestations. Lorsque la compagnie de Danse SeraQ, de Belo Horizonte (Minas Gerais, Brésil), menée par Rui Moreira se présente à Dakar au Festival mondial des arts nègres de 2010 avec son spectacle $Q$ eu isse, qui réinterprète une manifestation de PCI du village d'Acupe (Bahia) le Nego Fugido $^{15}$, non encore reconnue nationalement, elle permet à un public non initié de s'approcher de l'esthétique de cette manifestation. Rui Moreira reprend, en effet, la jupe faite de feuilles de bananier séchées et tressées, le torse nu, la langue rouge de colorant, propres au Nego Fugido, qu'il revisite sur une musique de samba sur laquelle se meuvent ses danseurs dans un décor très contemporain. Cette installation plastique faite d'un amoncellement labyrinthique de cartons en équilibre instable accueille la transe de Rui Moreira, qu'il reprend également de la manifestation. Tel un Derviche Tourneur, Rui tourne et «s'envole » au milieu des cartons et s'arrête enfin plaqué au sol. Il le fait sur la musique de la samba de Rio de Janeiro, reconnue PCI national par l'Iphan depuis $2007^{16}$. Souhaite-t-il de cette manière rendre cette musique décalée en présence de représentations du Nego Fugido, pour faire un parallèle politique entre la lutte des anciens esclaves et la situation des Afro-Brésiliens en homogénéisant une situation à tout le Brésil, ou pour interroger cette mainmise de la samba sur l'identité nationale ?

Une autre artiste, performeuse, Maria-Eugenia Vitali Manfredini, à l'inverse, utilise au sein de sa performance «Marias, Maria, ou Joana ? de la musique classique de Tchaikovsky Le lac des cygnes, dans une panoplie complète de danseuse de samba, incluant plumes, talons hauts, string, paillettes, diadème. Elle s'est présentée en France en octobre 2015 et interroge tout d'abord la domination de la danse classique en arts chorégraphiques au Brésil, dans les écoles de danse et même à l'université. En effet, dans les départements de danse et de théâtre des universités brésiliennes, il existe très peu de cours de manifestations issues des différents patrimoines immatériels, ce qui porte préjudice à la transmission de ces savoirs auprès des nouvelles générations d'artistes. De plus, Maria-Eugenia s'interroge sur le lien « race genre » au travers de la samba. Elle considère la samba

15. Le Nego Fugido a lieu chaque année à Acupe, en juillet, durant trois week-ends et se joue dans les rues du village. La manifestation réinterprète l'esclavage et son abolition en 1888 par la Princesse Isabelle. Les anciens esclaves ont le visage peint en noir, les gardiens d'esclave ont la jupe en feuille de bananier évoquée ci-dessus. La musique jouée reprend les rythmes du candomblé, exceptionnellement rendus profanes pour cette manifestation, ce qui favorise, néanmoins, l'induction de la transe. Rui Moreira, en décidant d'extraire certains éléments de cette manifestation donne un contenu engagé en faveur de la revalorisation des Afro-Brésiliens au sein de la société brésilienne, mais permet également de transmettre un savoir, celui de l'esthétique de ce patrimoine immatériel méconnu tant au Brésil qu'à l'étranger.

16. Autour de Rui Moreira, voir: http://www.centroculturalvirtual.com.br/noticias (consulté le 10 novembre 2015). 
comme une danse stéréotypée concernant la place de la femme afro-brésilienne tant à l'étranger que nationalement : la danseuse de samba mulâtre en tant qu'objet sexuel ${ }^{17}$.

Danseuse de formation, elle interprète dans sa performance la musique classique sur des pas de danse de samba, et petit à petit, simule une transe entre possession d'une orixá et d'une poule. Puis elle se change derrière un paravent et revient toute vêtue en blanc, tête couverte d'un long tissu blanc, à l'instar des officiants de candomblé, distribuant des œufs durs comme autant d'offrandes rituelles ${ }^{18}$. Elle danse au sol et se contorsionne sur un second morceau de musique classique. Elle détourne le savoir chorégraphique dominant lié à la danse classique en se l'appropriant nationalement au travers de la samba carioca, symbole national et PCI reconnu, puis en détournant ce savoir au profit du candomblé, comme véritable représentant de l'identité afro-brésilienne positive, car non-emprunt des stéréotypes de la mulâtre de la samba, mais représentant des «femmes fortes ». Elle se détourne donc des «Beaux-Arts » issus de l'art occidental, pour questionner ici la danse classique, puis interroge le fait que la samba soit, d'une certaine façon, aussi stéréotypée que la danse classique et cherche dans une manifestation immatérielle « afro » un autre visage pour une nouvelle esthétique nationale dans la lignée des pères du mouvement noir dans les années 1940.

\section{Conclusion}

La capacité de reconnaissance et d'ascension sociale des détenteurs de patrimoines immatériels rendue possible grâce à une politique publique volontariste initiée par le ministre de la Culture du premier gouvernement Lula, Gilberto Gil, aujourd'hui controversée, est intimement liée à la transmission des savoirs issus de ces manifestations ${ }^{19}$. Cette transmission en direction tant du grand public que des communautés détentrices se révèle polymorphe. Elle passe par les différents plans de sauvegarde mis en place par les institutions ou les associations qui en ont la responsabilité et favorisent l'usage des savoirs patrimoniaux par tous et plus particulièrement pour les jeunes générations. Ces dernières les font évoluer, les hybrident et les adaptent au sein, ou non, des communautés.

Pourtant, la question de déterminer à quel public s'adresse le savoir issu du PCI n'est pas clairement résolue ni auprès des détenteurs de ce savoir ni auprès des instigateurs des politiques publiques qui fomentent conjointement les plans de sauvegarde et gèrent les subventions. Pour les promoteurs des plans de sauvegarde, il n'existe pas, a priori, de savoir endogène à la communauté détentrice du PCI. Si des CDs de musique - enregistrés par les détenteurs des patrimoines

17. Entretien avec M. E. Vitali Manfredini, à Besançon, le 10 novembre 2015.

18. Les œufs crus sont très présents dans le candomblé.

19. Il reste à déterminer quelle sera la pérennité de cette impulsion politique à la suite du durcissement des politiques publiques depuis 2016 et quels moyens les communautés détentrices parviendront à mettre en œuvre pour la substituer. 
immatériels eux-mêmes - circulent, si des personnes extérieures à la communauté s'approprient la musique ou la danse grâce à des cours distillés par les communautés, une limite à la transmission du savoir et une échelle hiérarchique des groupes fondée sur leur «authenticité » semblent peu pertinentes pour les instigateurs des plans de sauvegarde. Le succès de telles politiques semble - à termes - transformer un PCI en un «savoir commun" à tous, « un bien commun » au risque d'une sensation de perte de légitimité pour ses premiers détenteurs vis-à-vis du reste de la société. Faut-il que les détenteurs du PCI déterminent eux-mêmes les règles de transmission de leur savoir au public exogène à la communauté ? La volonté de contrôle du savoir de la part de la communauté détentrice du PCI est une tentation tant sur le plan symbolique que sur le plan matériel puisque ces communautés tirent une partie de leurs ressources de ce PCI.

Néanmoins, malgré ces appréhensions émanant des communautés détentrices du savoir, la transmission s'opère également par une nouvelle relation au monde de l'art. D'ailleurs, ce rapport à l'art s'est peu à peu mis en place depuis les années 1930. Il est à la fois lié aux nouvelles revendications nationales en Amérique latine, mais également aux questionnements touchant l'ensemble de l'art contemporain à cette période, à l'instar du mouvement primitiviste. Ainsi, les savoirs des patrimoines immatériels en tant que «signaux à coût élevé » sont transmis par le biais d'une appropriation de ces savoirs au sein d'autres domaines de création contemporaine ou bien sont eux-mêmes hissés au statut d'œuvre d'art. Une telle pratique d'appropriation des savoirs des PCI au sein des créations contemporaines semble favoriser la pérennisation des savoirs immatériels, mais peut nuire à la créativité des artistes si cette démarche devient systématique. En effet, les artistes risquent de limiter leur horizon créatif à une demande d'exotisme stéréotypé, souvent associé à un PCI et potentiellement réducteur, de la part du marché de l'art. Ce dernier, ainsi que l'institution muséale constituent bien souvent une « usine à clichés » [Vincent, 2015, p. 203].

Mais comme le montre Dominique Malaquais ${ }^{20}$ - au sujet du sculpteur camerounais Hervé Youmbi - il est possible de faire du « faux traditionnel » en reprenant la technique de perles des masques du Grassfield du Cameroun, qui est un PCI très prisé sur le marché de l'art, et d'y inscrire le logo de Nike ou la marque de Superman. Peuvent-ils encore être identifiés comme des masques du Grassfield? Ils sont simplement des créations plastiques contemporaines, mais qui mobilisent et hybrident des savoirs patrimoniaux de telle sorte qu'ils en proposent une forme de vie. De la même manière, lorsque dans les années 1990, Chico Science (Nação Zumbi) intègre le rythme du maracatu et qu'il crée le Mangue Beat, il s'approprie les racines de ce patrimoine afro-brésilien et le transforme en une musique contemporaine. Le maracatu, malléable, semble ici être devenu un bien commun, véritablement populaire. Chico Science a permis, jusqu'à

20. Conférence de D. Malaquais au sein du séminaire « Supports et circulation des savoirs et des arts », EHESS, le 27 mars 2015. 
aujourd'hui, un renouveau de la musique du Nordeste (Pernambuco notamment dont il était originaire) et une forte collaboration entre jeunes musiciens et maîtres de patrimoines immatériels.

\section{Bibliographie}

Alencar R., BandeIRa R. [2010], O Samba de Roda na gira do patrimonio, doctorat en anthropologie, Unicamp, Brésil.

Abreu R., Chagas M. (dir.) [2003], Memória e patrimônio, Rio de Janeiro, Lamparina.

Amoroso D. [2011], « O samba de roda enquanto obra-prima do patrimônio cultural imaterial : questões, impactos e perspectivas », conférence au VII Cinecult, Salvador, Bahia, 3 au 5 août 2011.

Amoroso D. [2009], Levanta mulher e corre a roda : dança, estetica e diversidade no Samba de Roda de São Felix e Cachoeira, doctorat en arts scéniques, universidade federal da Bahia, Salvador.

ANDRADE M. (de) [2002], «Anteprojeto para a criação do serviço do patrimônio artístico nacional » (datant de 1936), Patrimônio histórico e artístico nacional, ${ }^{\circ}$ 30, p. 129-136.

AsSociaÇão dos SAMBADORES E SAMBADEIRAS DO ESTADO DA BAHIA (ASSEBA) [2015], As mulheres do Samba de Roda (documentaire), Rede do Samba de Roda do Recôncavo Baiano, chegança dos marujos fragata brasileira, IPAC-Fundo de Cultura-Governo da Bahia, Salvador.

Barbosa F., Calabre L. (dir.) [2011], Pontos de cultura : olhares sobre o programa cultura viva, Brasília, Ipea.

Belas C. A. [2004], « Aspectos legais do instituto do iventario nacional de eferências culturais (INRC), Relação com legislações nacionais e acordos internacionais », Patrimônio histórico e artístico nacional-Iphan, $\mathrm{n}^{\circ} 31,27 \mathrm{p}$.

Belas C. A. [2004], «A Propriedade intelectual no âmbito dos direitos difusos », in TeIXeIRA J. G. L. C., Garcia M. V. C., Gusmao R. (dir.), Patrimônio imaterial, performance culturale $e$ (re) tradicionalização, Brasília, ICS-UnB.

Bortolotto C. [2007], «From objects to processes : Unesco's intangible cultural heritage », Journal of Museum Ethnography, n 19, p. 21-33.

Bortolotto C. (dir.) [2011], Le Patrimoine culturel immatériel. Enjeux d'une nouvelle catégorie, Paris, Maison des sciences de l'homme.

CAPONE S., RAmos de Morais M. [2015], « Afro-patrimoines, cultures afro-brésiliennes et dynamiques patrimoniales », Les cahiers du Lahic, $\mathrm{n}^{\circ} 11,303 \mathrm{p}$.

Cavalcanti M. L. [2001], « Cultura e saber do povo : uma perspectiva antropologica », Tempo brasileiro, $\mathrm{n}^{\circ} 147$, p. 69-78.

CiARCIA G. [2016], Le revers de l'oubli. Mémoires et commémorations de l'esclavage au Bénin, Paris, Karthala.

Celius C. A. [2015], «Quelques aspects de la nouvelle scène artistique d'Haïti », Gradhiva, $n^{\circ} 21$, p. 104-129.

Celius C. A. [2005], « Cheminement anthropologique en Haïti », Gradhiva, no 1, p. 47-55.

Claessen H. J. M. [2002], « “Comments” on Nas'article », Current anthropology, vol. 43, n 1, p. 144. 
Chuva M. [2009], Os arquitetos da memória: sociogênese das práticas de preservação do patrimônio cultural no Brasil (anos 1930-1940), Rio de Janeiro, université fédérale de Rio de Janeiro.

Deacon H., Luvuyo D., Mbulelo M., Prosalendis S. [2004], The subtle power of intangible heritage : legal and financial instruments for safeguarding intangible heritage, Capetown, HSRC Publishers.

De Jong F., Rowlands M. (dir.) [2007], Reclaiming heritage. Alternative imaginaries of memory in West Africa, Walnut Creek (C. A.), Left Coast Press, 270 p.

DörING K. (dir.) [2014], Cartilha do Samba chula, Bahia, UPB-União dos municípios da Bahia.

Douxami C. [2010], «Danse-théâtre et anthropologie. Un rapport dialogique entre soi et l'autre », Études théâtrales, n 49, p. 191-198.

DouxAmi C. [2015], «La politique de patrimoine culturel immatériel au Brésil : une volonté politique de démocratisation et d'inclusion des "minorités" ", in CAPONE S., RAMOS DE MoraIs M. (dir.), «Afro-patrimoines. Culture afro-brésilienne et dynamiques patrimoniales », Les carnets du Lahic, $\mathrm{n}^{\circ} 11$, p. 77-97.

FALCÃo J. [2001], « Patrimônio imaterial : um sistema sustentável de proteção », Tempo, n ${ }^{\circ}$ 147, p. $163-180$.

FonseCA M. C. L. [1997], O Patrimônio em processo : trajetória da politica federal de preservação no Brasil, Rio de Janeiro, université fédérale de Rio de Janeiro, ministère de la Culture-Iphan.

GonÇALVES J. R. S. [1995], A Retórica da perda : os discursos do patrimônio cultural no Brasil, Rio de Janeiro, université fédérale de Rio de Janeiro, Iphan.

HAFSTEIN V. T. [2007], "Claiming culture : intangible heritage Inc., folklore, traditional knowledge », in Hemme D., TauscheK M., Bendix R. (dir.), Prädikat "Heritage” - perspektiven auf wertschöpfungen aus kultur, Münster, Lit Verlag.

HyLland ERIKSEN T. [2001], «Between universalism and relativism : a critique of the Unesco Concept of Culture » in Cowan J. K., Dembour M. B., Wilson R. A., Culture and Rights : Anthropological Perspectives, Cambridge, Cambridge University Press, p. 127-148.

Jeudy H. P. [2001], La Machinerie patrimoniale, Paris, Sens \& Tonka.

JOLHER R. [2002], « The EU as manufacturer of tradition and cultural heritage », in KocKEL U. (dir.), Culture and Economy: Contemporary Perspectives, Ashgate, Aldershot.

Kirshenblatt-Gimblett B. [2004], From ethnology to heritage : the role of the Museum, Marseilles, Sief Keynote.

LAUDE J. [2006], La peinture française et «l'art nègre » (1905-1914). Contribution à l'étude des sources du fauvisme et du cubisme, Paris, Klincksieck.

LEIMGRUBER W. [2010], «Switzerland and the Unesco convention on intangible cultural heritage », Journal of Folklore Research, vol. 47, $\mathrm{n}^{\circ}$ 1-2, p. 161-196.

Maguet F. [2011], «L'image des communautés dans l'espace public », in Bortolotto C. (dir.), Le Patrimoine culturel immatériel. Enjeux d'une nouvelle catégorie, Paris, Maison des sciences de l'homme.

Ministerio de CUltura [S. D.], Plano national de culture : http://pnc.culturadigital.br/metas/ 15-mil-pontos-de-cultura-em-funcionamento-compartilhados-entre-o-governo-federal-asunidades-da-federacao-ufs-e-os-municipios-integrantes-do-sistema-nacional-de-cultura-snc/ (consulté le 2 mai 2015). 
Mompoint M. [2008], Symbolic exchanges: Haiti, Brazil and the Ethnopoetics of Cultural Identity, open access dissertations, paper 108, University of Miami : https://scholarlyrepository.miami.edu/oa_dissertations/108 (consulté le 2 mai 2015).

NAS P. J. M. [2002], « Masterpeaces of oral and intangible culture : reflexions on the Unesco world heritage list », Current Anthropologie, vol. 43, n 1, p. 139-148.

Nora P. [1984], Les Lieux de mémoire, La République, tome 1, Paris, Gallimard.

Nora P. [1986], Les Lieux de mémoire, La Nation, tome 2, Paris, Gallimard.

Nora P. [1992], Les Lieux de mémoire, Les France, tome 3, Paris, Gallimard.

Parise N. [2014], «Rencontre de Macunaïma et de l'Oncle », in PiCARd Byron J. (dir.), Production du savoir et construction sociale, l'ethnologie en Haïti, Québec/Port-au-Prince, Presses de l'université Laval/Presses de l'université d'État de Haïti, p. 181-197.

PENTEAdO W. J.-R. [2015], «Entre voix, énigmes et regards : le processus de reconnaissance patrimoniale du jongo et la "nation brésilienne" «, in CAPONE S., RAMOS DE MoraIs M., «Afro-patrimoines. Culture afro-brésilienne et dynamiques patrimoniales », Les carnets $d u$ Lahic, $\mathrm{n}^{\circ} 11$, p. 119-138.

Pinheiro BRessan M. L. [2006], «Origens da noção de preservação do patrimônio cultural no Brasil », Risco, ${ }^{\circ} 3$, p. 4-14.

SANDRONI C. [2011], «L'ethnomusicologue en médiateur du processus patrimonial : le cas de la Samba de Roda » in Bortolotto C. (dir.), Le Patrimoine culturel immatériel. Enjeux d'une nouvelle catégorie, Paris, Maison des sciences de l'homme.

SANDRONI C. [2010], «Samba de roda, patrimônio imaterial da humanidade », Estudos avançados, vol. 24, nº 69, p. 9.

SAnT'Anna M. [2001], « Patrimônio imaterial do conceito ao problema da proteção », Tempo brasileiro, $\mathrm{n}^{\circ} 147$, p. 151-161.

Sant' Anna M., SANDroni C. [2007], Samba de Roda no recôncavo bahiano, Brasilia, Iphan.

SHAEFFER J.-M. [2008], « Le musée du quai Branly entre art et esthétique », Le Débat, nº 148, p. $170-178$.

SCHECHNER R. [1988], Performance Theory, New York, London, Routledge.

SCHECHNER R. [1985], Between Theatre and Anthropology, Philadelphia (Pa.), University of Pennsylvania press.

StoczKowski W. [2009], « Unesco's Doctrine of Human Diversity : a Secular Soteriology ? », Anthropology Today, vol. 25, n 3, p. 7-11.

SMITH L. [2006], Uses of Heritage, London, Routledge.

Smith L., Akagawa N. (dir.) [2008], Intangible heritage, Oxford, Routledge.

Sylla A. [1999], «Présence africaine et art nègre », Présence africaine, 50 anniversaire, numéro spécial, p. 291-325.

TAYLOR D. [2008], «Performance and intangible cultural heritage », in DAVIS T. C., The Cambridge companion to performance studies, Cambridge, Cambridge Université Press.

TelLEs M. F. de P. [2007], «O registro como forma de proteção do patrimonio cultural imaterial », Revista $C P C, \mathrm{n}^{\circ} 4$, p. 40-71.

Tornatore J.-L. [2010], « L'esprit du patrimoine », Terrain, no ${ }^{\circ}$ 55, p. 106-127.

TURNER V. [1974], Dramas, fields, metaphors. Symbolic action in human society, Ithaca (N. Y.), Cornell University Press.

TURNER V. [1987], The Anthropology of performance, New York, PAJ Publication. 
UNESCO [2003], Convention for the safeguarding of the intangible cultural heritage, General provisions, Paris, Unesco.

UnESCO [S. D.], Samba de Roda of the Recôncavo of Bahia: www.unesco.org/culture/ich/ index.php?lg=en\&pg=00011\&RL=00101 (consulté le 4 novembre 2016).

Veloso M. M. S. [1992], O tecido do tempo : a ideia de patrimônio cultural no Brasil, 1920-1970, thèse d'anthropologie, université de Brasília, Brasília.

VIANNA L. [2004], « Patrimônio imaterial : legislação e inventários culturais », in LONDRES C., Carvalho L., Pacheco G., Nogueira V., Dina M., Mendonça E., Travassos E., CARVAlho (de) J. J., Celebrações e saberes da cultura popular : pesquisa, inventário, crítica, perspectivas, Rio de Janeiro, Funarte, Instituto do Patrimônio histórico e artístico national (Iphan), Centro nacional de Folclore e Cultura populaire (Cnfcp), encontros e estudos $n^{\circ} 5$.

VINCENT C. [2015], « Réinventer le musée : interactions et expérimentations », Perspective, $\mathrm{n}^{\circ} 1$, p. 199-206. 